\title{
RESONANT BEAM RESPONSE IN THE PSR ACCUMULATOR RING*
}

\author{
J.A. Holmes, V. Danilov, J.D. Galambos, SNS, ORNL, Oak Ridge, TN 37831-6473, USA \\ A. Fedotov, BNL, Upton, NY 11973, USA \\ R. Gluckstern, University of Maryland, College Park, MD 20742, USA
}

\begin{abstract}
Observed beam profile broadening at high intensity in the Proton Storage Ring (PSR) at Los Alamos National Laboratory motivates this analysis of resonant beam response using a particle core model. Previous numerical PIC code simulations gave good agreement with the measured beam profiles, and analysis of those simulations suggested that the profile broadening at high beam intensities is related to the half integer resonance at $Q_{\mathrm{y}}=2$ (the bare tune was $Q_{\mathrm{y}}=2.14$ ). The physics of half integer resonances has been studied analytically using the envelope equations. We now carry out particle core model calculations for the PSR lattice under the same conditions as the previous PIC calculations. We show that the resonant behavior of the envelope equations is consistent with the previous PIC and experimental findings and with the theoretical envelope equations analysis for half integer resonances. These results provide further support for the half integer resonance at $Q_{\mathrm{y}}=2$ as the cause of the beam broadening.
\end{abstract}

\section{INTRODUCTION}

We analyze beam profile broadening at high intensity in the Proton Storage Ring (PSR) at Los Alamos National Laboratory using a particle core model. Previously, beam profile measurements were performed in the extraction line for a range of intensities and then simulated in detail numerically using the PIC code, ORBIT [1]. In particular, careful attention was paid to lattice settings, injection, painting, and RF scenarios. These simulations gave good agreement with the measured beam profiles [2], both showing a systematic broadening in the vertical direction at high intensities. Subsequent analysis of the simulations suggested that the profile broadening is related to the half integer resonance at $Q_{\mathrm{y}}=2$ (the bare tune was $Q_{\mathrm{y}}=2.14$ ) [3]. The simulations indicated that the beam broadening at high intensity is associated with the incoherent tunes of a substantial fraction of the beam crossing $Q_{\mathrm{y}}=2$. Furthermore, the simulations showed that the adopted injection and $\mathrm{rf}$ scenarios resulted in longitudinally peaked bunches, with much higher densities in the bunch center than at the ends, and also that that both tune shifts and beam broadening were greater at the bunch center than near the ends. Spectrum analysis of the beam second moments in the simulations shows a large $\mathrm{N}=4$ component at high intensity, consistent with a coherent half integer resonance at $Q_{\mathrm{y}}=2$. Finally, the RMS emittance was observed to increase with the amplitude of the $\mathrm{N}=4$ component of the second moment above a threshold value, which is also consistent with a coherent half integer resonance at $Q_{\mathrm{y}}=2$.

The physics of half integer resonances was studied analytically using the transverse envelope equations by Sacherer in his $\mathrm{PhD}$ thesis [4]. These equations can be written

$$
\begin{aligned}
\frac{d^{2} y}{d \phi^{2}}= & -\left[Q_{y}^{2}+2 Q_{y} \cdot \Delta Q_{e y} \cos n \phi\right] y+\frac{Q_{y}^{2}}{y^{3}} \\
& +\frac{4 r_{0} \lambda}{\gamma^{3} \beta^{2}} \frac{Q_{y}^{2} \beta_{y}^{3 / 2}}{\sqrt{\varepsilon_{y}}} \frac{1}{\sqrt{\beta_{x} \varepsilon_{x}} x+\sqrt{\beta_{y} \varepsilon_{y}} y}
\end{aligned}
$$

and similarly for $\mathrm{x}$, where $\mathrm{y}$ is dimensionless and given by $y \rightarrow \frac{y}{\sqrt{\beta_{y} \varepsilon_{y}}}, \phi$ is the longitudinal coordinate given by $d \phi=\frac{d s}{Q_{y} \beta_{y}}, Q_{y}$ is the bare tune, $\Delta Q_{e y}$ is the quadrupole field error of periodicity $\mathrm{n}, \lambda$ is the longitudinal particle density, $\beta_{x, y}$ are the beta functions, and $\varepsilon_{x, y}$ are the rms emittances. In solving the envelope equations for periodic solutions, Sacherer made two simplifying assumptions: 1 ) he ignored the variation of the beta functions and used instead their average values, and 2) he assumed both the magnet error and space charge terms were small and accordingly applied a perturbation approach. Sacherer's analysis makes two predictions that are important for PSR: 1) he predicts that the coupled $\mathrm{x}$ and $\mathrm{y}$ envelope equations act independently unless the incoherent tune are nearly equal, and 2) he predicts that it is possible for the incoherent tunes to cross the half integer values to some degree before the resonance is excited. To quantify these predictions, Sacherer finds for circular beams that strong coupling occurs only when $Q_{x}-Q_{y}<\frac{\Delta Q_{s c}}{4}$, where $Q_{s c}$ is the space charge tune shift. For PSR at high intensity, $Q_{s c} \approx 0.2$, and with $\left(Q_{x}, Q_{y}\right)=(3.17,2.14)$ weak $\mathrm{x}$-y coupling is anticipated. In this case, the coupling of the envelope equations is weak, and these results state that the half integer resonance will occur when the space charge tune shift satisfies $\Delta Q_{s c}=\frac{8}{5}\left(Q_{y}-R\right)$, where $R$ is the half integer value 
associated with the resonance. Thus, for PSR with bare tune $\mathrm{Q}_{\mathrm{y}}=2.14$, the resonance is predicted to occur at $\Delta Q_{s c}=\frac{8}{5}\left(Q_{y}-2\right)=0.224$, so that the corresponding incoherent tune is $Q_{i y}=1.916$. The calculations carried out here use the PSR lattice and settings, but they do not include quadrupole strength errors. In this case, the term $\Delta Q_{e y}=0$, and the resonance is predicted to be narrow, with constant solutions $y=1$ away from the resonance.

Motivated by Sacherer's analysis, we now carry out particle core model calculations for the PSR with the same lattice settings as the previous PIC calculations. Unlike Sacherer, we solve the full, coupled envelope equations numerically without approximations. We show that the envelope equations resonate at beam intensities consistent with the profile broadening in PSR and at incoherent tunes consistent both with the profile broadening seen in the PIC simulations and with the analytic treatment of Sacherer. These results provide further support for the half integer resonance at $Q_{\mathrm{y}}=2$ as the cause of the beam broadening.

\section{RESULTS}

Previous PIC simulations incorporated the actual PSR injection scenarios with painting and bunched beams. In these cases, the bunch factors were significantly different from unity and the beam spreading was strongest in the vicinity of peak bunch intensity. Because the envelope equations are solved for a uniform-density coasting beam, it is appropriate to compare the PIC results with envelope equation simulations at beam intensities corresponding to the peak longitudinal densities in the PIC simulations. Figure 1 facilitates this comparison by plotting the number of protons and the peak beam intensity (number of protons / bunch factor) in a high intensity simulation as a function of turn number. We emphasize that the blue, not the red, curve shows the actual number of protons in the bunch. In the PIC simulation, noticeable beam broadening began between 700 and 1000 turns and increased until the end at 2864 turns.

In the present envelope equation calculations, periodic solutions were found for the PSR lattice with bare tunes of $\left(Q_{x}, Q_{y}\right)=(3.17,2.14)$ and beam intensities varying from $0 \rightarrow 2 \cdot 10^{14}$ protons. For each of these cases, the incoherent tunes of test particles placed inside the space charge potential of the uniform density envelope were calculated. Figure 2 plots the incoherent tune $Q_{\text {iy }}$ as a function of beam intensity for these calculations. The curve reveals a broad bump for intensities between $5 \cdot 10^{13} \rightarrow 1.2 \cdot 10^{14}$ protons and corresponding $1.96 \geq Q_{i y} \geq 1.7$. Comparison with Figure 1 places these intensities from about 1000 turns through the end of the run, precisely during the observed beam broadening.

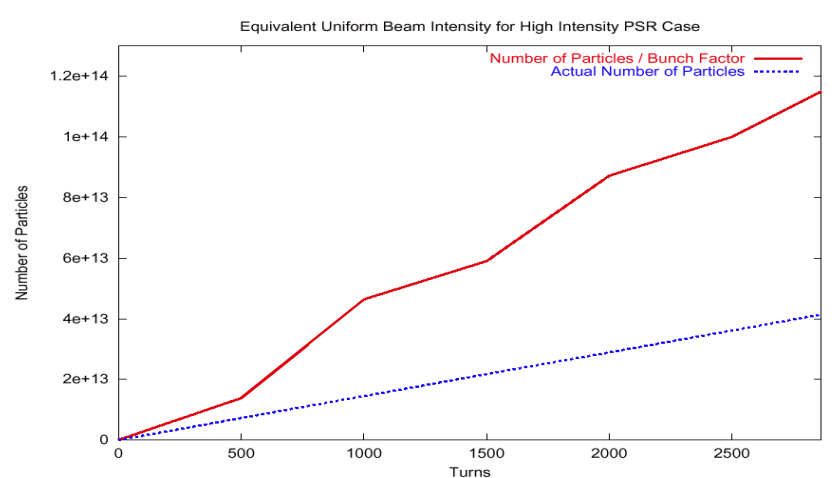

Figure 1. Actual and peak beam intensities versus turn number for high intensity PSR simulation.

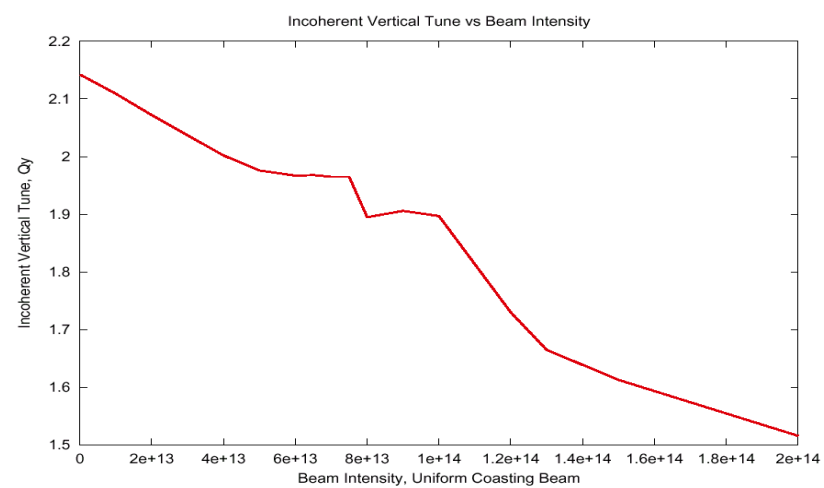

Figure 2. Incoherent tune $Q_{\text {iy }}$ versus beam intensity.

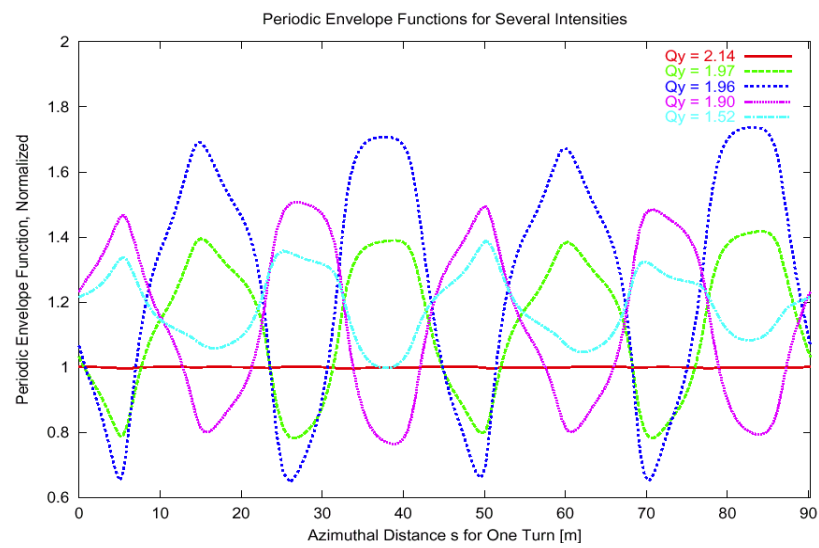

Figure 3. Periodic envelope functions for various beam intensities.

The periodic solutions of the envelope equations are plotted for several beam intensities as functions of the distance around the PSR ring in Figure 3. For zero intensity, $Q_{i y}=2.14$, the envelope function is constant with value unity. As intensity increases, so does the oscillation amplitude, until it peaks at $Q_{i y}=1.96$. For higher intensities (lower incoherent tunes) the oscillation amplitude decreases and flips sign. Figure 4 shows the amplitude of the envelope functions plotted versus beam intensity. There is a strong peak at $7.5 \cdot 10^{13}$ protons, which corresponds to the intensity at about 1750 turns in 
the PIC simulation shown in Figure 1. Figure 5 plots the amplitude instead as a function of the incoherent tune, $Q_{\mathrm{iy}}$. Starting at the bare tune, the amplitude increases as the incoherent tune decreases below 2.0, and peaks strongly at $Q_{i y}=1.96$ before dropping off at lower $Q_{\mathrm{iy}}$. The resonance peak is not narrow as in Sacherer's onedimensional analysis and the envelope amplitudes do not return to unity at high intensities beyond the resonance peak. Also, solutions for the horizontal envelope function reveal a weaker dependence of amplitude on intensity, consistent with the theoretical finding of weak $x-y$ coupling.

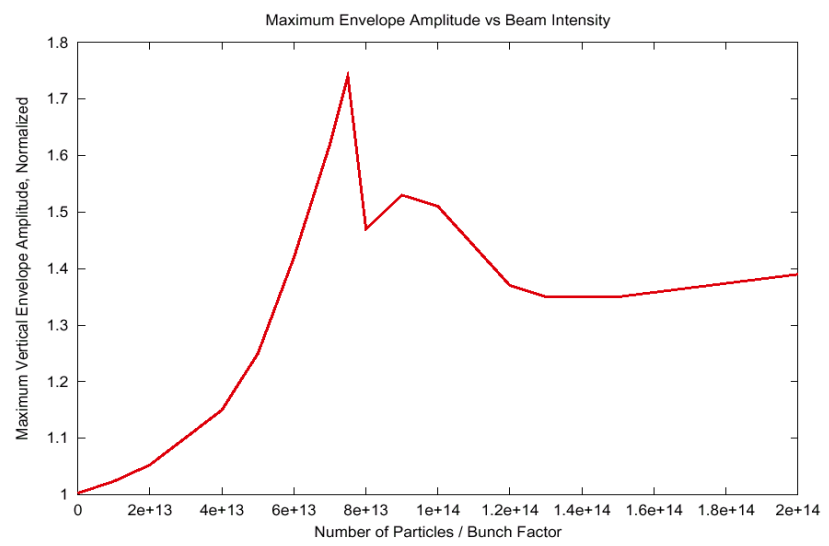

Figure 4. Amplitude of periodic envelope function versus beam intensity.

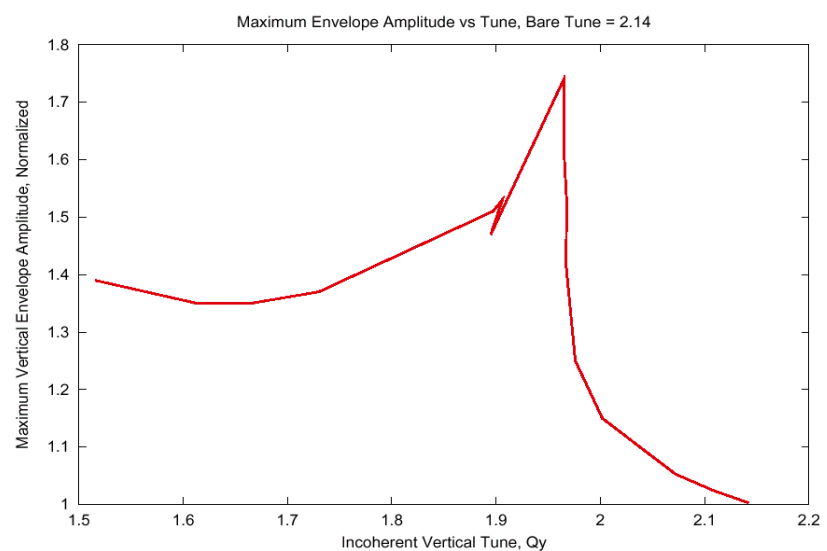

Figure 5. Amplitude of periodic envelope function versus incoherent tune.

\section{DISCUSSION}

The results of these calculations of periodic solutions of the full, coupled envelope equations in the PSR lattice are consistent with previous measurements and PIC simulations. The calculations show a bump in the incoherent tune function and a strong peak in the envelope amplitudes at beam intensities comparable to those for which beam broadening was observed. The bump in the plot of the tunes is a nonlinear effect that is strongly related to the envelope amplitude fluctuations. For large amplitude fluctuations, space charge forces are, on average, weaker and lead to smaller tune shifts, or higher tune values. In fact, if one "removes the bump" from the tune plot in Figure 2 by connecting the low and high intensity curves continuously, Sacherer's predicted value of $Q_{i y}=1.916$ for the resonance occurs somewhat above $6 \cdot 10^{13}$ protons, or at 1500 turns according to Figure 1. This is well within the resonance, and the peak of the amplitude function occurs at $7.5 \cdot 10^{13}$ protons, just before dropping out of resonance and into the high intensity behavior, which is indicated by the sign reversal of the envelope oscillations in Figure 3. The resonance peak appears above Sacherer's predicted value of the incoherent tune in Figure 5 because the incoherent tune scale is affected, as shown in Figure 1, by the large envelope oscillations in the resonance.

Furthermore, the results are consistent with Sacherer's theoretical analysis in that the $\mathrm{x}$-y coupling is weak for these tunes. It is also interesting that the periodic envelope oscillations change character, flipping sign, as the resonance is crossed. Only the width of the resonance peak and the high intensity behavior of the amplitudes differ from the theoretical analysis, and these differences are not surprising, given the assumptions of averaged beta functions and small space charge forces used in the theory.

Thus, the present results, coupling experiment, PIC simulations, and analytic calculations through solution of the envelope equations lend further support to the picture of the high intensity PSR beam broadening as the manifestation of a half integer resonance at $Q_{\mathrm{y}}=2$.

\section{ACKNOWLEDGEMENTS}

We wish to thank Bob Macek and the PSR staff for encouraging this work and for providing access and assistance in carrying out high intensity beam profile measurements at PSR.

Research on the Spallation Neutron Source is sponsored by the Division of Materials Science, U.S. Department of Energy, under contract number DE-AC05-96OR22464 with UT-Battelle Corporation for Oak Ridge National Laboratory.

\section{REFERENCES}

[1] J. Galambos, J. Holmes, D. Olsen, A. Luccio, and J. Beebe-Wang, ORBIT Users Manual, http://www.sns.gov//APGroup/Codes/Codes.htm

[2] J. D. Galambos, S. Danilov, D. Jeon, J. A. Holmes, D. K. Olsen, F. Neri, and M. Plum, Phys. Rev. Special Topics - AB 3, (2000) 034201.

[3] J. A. Holmes, A. Aleksandrov, J. D. Galambos, D. K. Olsen, F. Merrill, and R. J. Macek, in Proceedings of the 2000 European Particle Accelerator Conference, (Vienna, 2000) 936.

[4] F.J. Sacherer, "Transverse Space-Charge Effects in Circular Accelerators," $\mathrm{PhD}$ Thesis, University of California, Berkeley (1968). 